\title{
Semantic Interrelation in Distributed System Through Green Computing Ontology
}

\author{
Herlina Jayadianti, Lukito Edi Nugroho, Paulus Insap \\ Santosa \\ Department of Electrical Engineering and Information \\ Technology \\ Universitas Gadjah Mada \\ Yogyakarta, Indonesia \\ herlinajayadianti@gmail.com, Lukito@ugm.ac.id, \\ insap@jteti.gadjahmada.edu
}

\author{
Carlos Alberta Baptista Sousa Pinto \\ Department of Information System \\ Universidade do Minho Guimaraes Portugal \\ csp@dsi.uminho.pt \\ Wahyu Widayat \\ Department Economic Development \\ Universitas Gadjah Mada \\ Yogyakarta, Indonesia \\ wahyu@mep.ugm.ac.id
}

\begin{abstract}
Green computing refers to the system that provides minimal impact on the environment. When we are talking about green computing we discuss about how much energy is used by the system, such as energy used by the system, time used for the search process, and how effective the system is. Related to that issue, trough this paper we want to proposes a new effort to achieve Green Computing in heterogeneous data in distributed system. The technology chosen to deal with them is Ontology. We try to generate a common ontology including a common set of terms, based on the several ontologies available, in order to make possible to share the common terminology (set of terms) that it implements, between different communities. If a very large amount of distributed data is not managed and distributed properly, user will need more time to do a search process. The longer the search is done, the more energy is used.
\end{abstract}

Keywords-Ontology; Green Computing, Data Heterogeneity; Effectiveness;

\section{INTRODUCTION}

Support of computer systems have become part of the national infrastructure of each country. Almost the entire national infrastructure has been utilizing computers to support and offer essential and critical services either distributed or not distributed. Problem then appeared if the required data are scattered and are in a place that is different, then of course search process would become longer and takes a significant level of electrical power, thus contributing to increased fuel consumption be. The idea of the green computing has become popular in recent concern, especially if it is linked to the issue of energy crisis. Green computing focuses on how much energy the system is used and how they can make it more efficient. Related to that issue, through this paper we would like to propose a new effort to achieve an efficient search process for distributed heterogeneous data [2].

A distributed system [5] is a collection of autonomous computers linked by a computer network that appear to the users of the system as a single computer. Design issues that arise specifically from the distributed nature of the application are: (1) Transparency, (2) Heterogeneity, (3) Performance, (4) Security, and (5) Openness. In this paper we will focus only in heterogeneity problem, such as: Technical heterogeneity, data model heterogeneity and semantic heterogeneity. Semantic heterogeneity is a general term referring to disagreement about the meaning, interpretation or intended use of the same or related data. This problem is poorly a clear definition of the problem [4], [6], [7]. The importance of being aware of semantic heterogeneity and doing semantic reconciliation is to guarantee meaningful data sharing. The technology chosen to deal with semantic heterogeneity problem is Ontology. Ontologies [8], [9], [10], [11] is a model for determining semantic concepts used by various heterogeneous sources in a way that clearly defined. As more ontologies are built in a different domain, ontology heterogeneity is become another significant problem for the integration of information.

Through this paper we want to prove that through ontology, can make the distribution of the data becomes easier without reducing the semantic meaning. We also want to propose a better solution in searching process to support an energy efficient [3], [18]. The objectives of this paper are to make an easy sharing semantic meaning; and to make the system can understand the tag given by each user. We will show the result trough a small implementation project. This paper is organized as follows: (1) Introduction; (2) Ontology and Green Computation; (3) Interrelation of Semantic Heterogeneity; (4) Implementation; (5) Discussion; (6) Conclusions of work.

\section{ONTOLOGY AND GREEN COMPUTATION}

Knowledge [1], [15] is people's individual map of the world. Knowledge acquisition involves complex cognitive processes such as perception, communication, and reasoning. According to the knowledge differences, then it is possible for people have a different perception to attain awareness or understand 
the environment or reality. There is a group of people in different communities managing data using different perceptions, different concepts, different terms (terminologies), and different semantics to represent the same reality. Perceptions are converted into data, and then saved into separate storage devices that are not connected to each other. Each user - belonging to different communities with large automatic environment - use different terminologies in collecting huge amount of data and as a consequence they also get different results of that exercise. It is not a problem if different results are used for each community. Problems occur if people need to take data from other communities and share it in a collaboration to get a better-quality solution. Ontology is a better technology to solve this problem. We can share different conceptualizations, different terminologies, and different meanings between systems using ontology. Ontology integration [14],[16],[17] and Ontology mapping [7],[8],[14] allows finding correspondences between the concepts of two or more ontologies. If two concepts correspond to each other, then they can also easily "understand" to each other. This is the main reason to bring ontology into green computing area. The effectiveness of search engine processing is closely associated with energy saving.

\section{INTERRELATION OF SEMANTIC HETEROGENEITY}

In this section, we will illustrate the problem of semantic heterogeneity and how to solve it. As an example there are different groups of people (different communities) have different set of term about object, example term "Hospital" in domain health. "Hospital" is a common term to refer a health facility where patients receive treatment or a medical institution where sick or injured people are given medical or surgical care, but it is not possible if people as an independent human being may use a different terms to refer term "hospital" such as "Infirmary", "medical building", "health facility", "healthcare facility", "clinic", "medical institution", or "extended care facility". (See Fig 1)

Even though each of people use a different "term" to refer "hospital" but in semantic have the same meaning (equivalent) e.g. term "Health facility" is equivalent to term "health care facility". People can easily argue and debate to each other about differences, but the ability of the human can not be replaced easily by computers. Computers need an artificial brain to understand the problems. It is a challenge that must be faced to make computers think like humans. (See Fig 2)

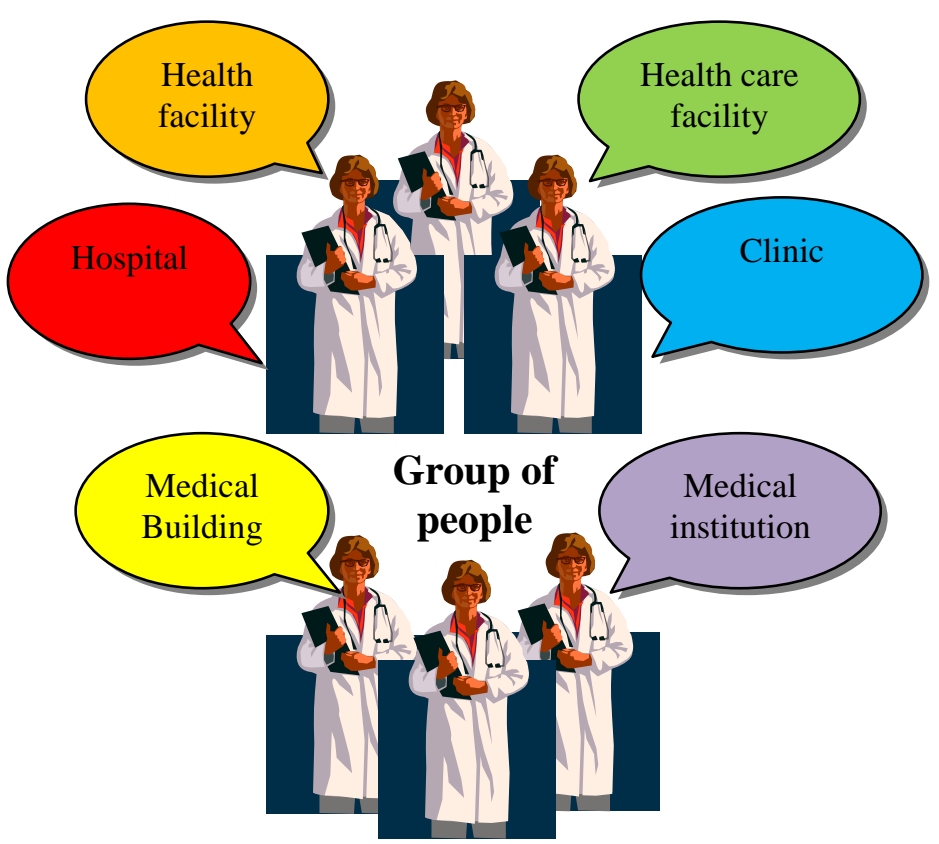

Fig 1. Group of people use different terms to refer the same object

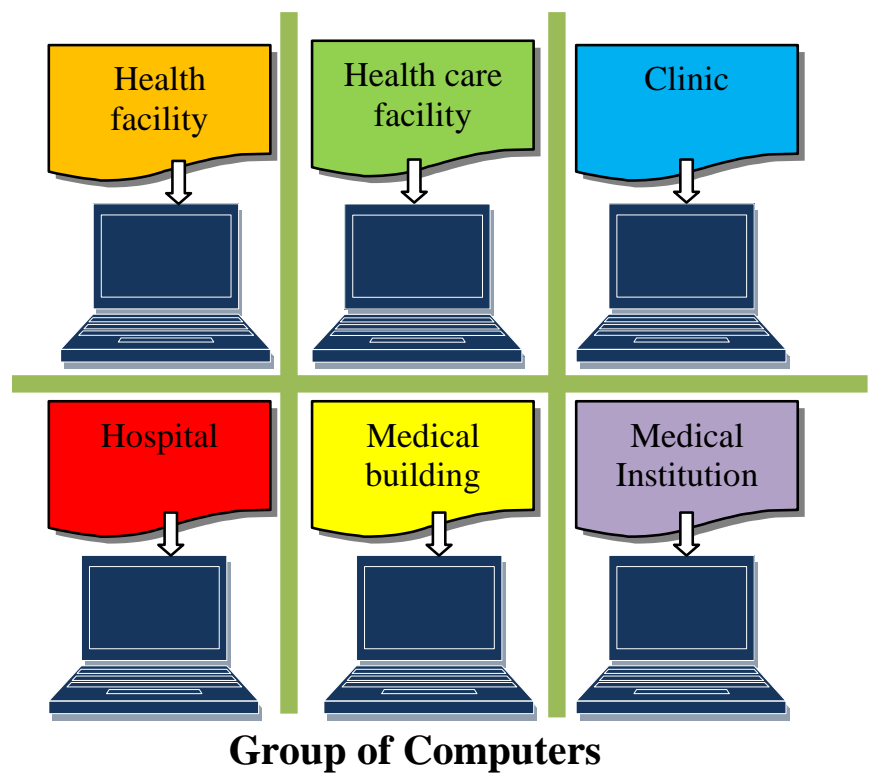

Fig 2. Different terms in different computers

Fig.2 represents several computers in different places that captured reality about the building where patients receive treatment. Let's make a scenario that one day a President as a user want to search data about Hospital in a country that he leads. In his knowledge and experience term "Hospital" is the only one term that he knows about the building where patients receive treatment. In reality each computer use different terms to refer the "building of treatment" (See Fig 2). As an example, one computer use term "Hospital" and another use term "Medical building". To be similar or not equal depend on several factors, such as the programmer's interpretation and the needs of the system itself. Users who 
deal with computers has a very important role in controlling and changing the terminology and semantic of the data. Let's see again Fig 1, if a group of people faced differences they will easily discuss and communicate to each other, but what happened if this situation appears in a group of computers. Knowledge representation and ontology are the technologies choose to solve this problem. First step, any data in each computer (Fig 2) are needed to be transferred into ontology (See Fig 3). Second step is mapping terms between ontology.

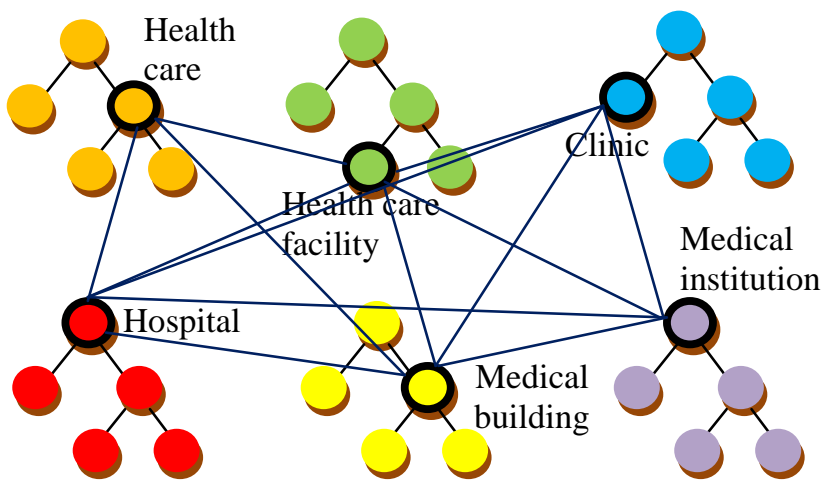

Fig 3. Semantic interrelation

Mapping [16] is the process of relating similar concepts or relations from different sources through some equivalence relation. Mapping allows finding correspondences between the concepts of two or more ontologies. If two concepts correspond, then they mean the same thing or closely related things e.g. "Hospital" from URI: http: //www. semanticweb.org/user/ontologies 1/\#Hospital EquivalentTo "HealthCare_facility" from URI:http://www.semanticweb.org/user/ontolog ies5\#Healthcare facility"/>, not only that term "Hospital" also EquivalentTo "Clinic", "Medical institution", "Medical building" and "Heal thCare". See the code in RDF/OWL below:

$<$ ?xml version="1.0"?>

http://www.semanticweb.org/user/ontologies1/\#Hospital $-->$

<rdfs:label xml:lang="en">Hospital</rdfs:label>

<owl : equivalentclass

rdf:resource="http://www.semanticweb.org/user/ontolog iesl\#Hospital"/>

<owl : equivalentClass

rdf: resource="http://www.semanticweb.org/user/ontolog ies2\#Medical_institution"/>

<owl: equivalēentclass

rdf: resource="http://www.semanticweb.org/user/ontolog ies3\#Infirmary"/>

<owl : equivalentClass

rdf: resource="http://www.semanticweb.org/user/ontolog ies4\#Medical building"/>

<owl : equivalēntclass

rdf:resource="http://www.semanticweb.org/user/ontolog ies5\#Healthcare_facility"/>

Ontology mapping allows finding correspondences between the concepts of two or more ontologies. Mapping illustration between ontologies is shows in Fig 4.
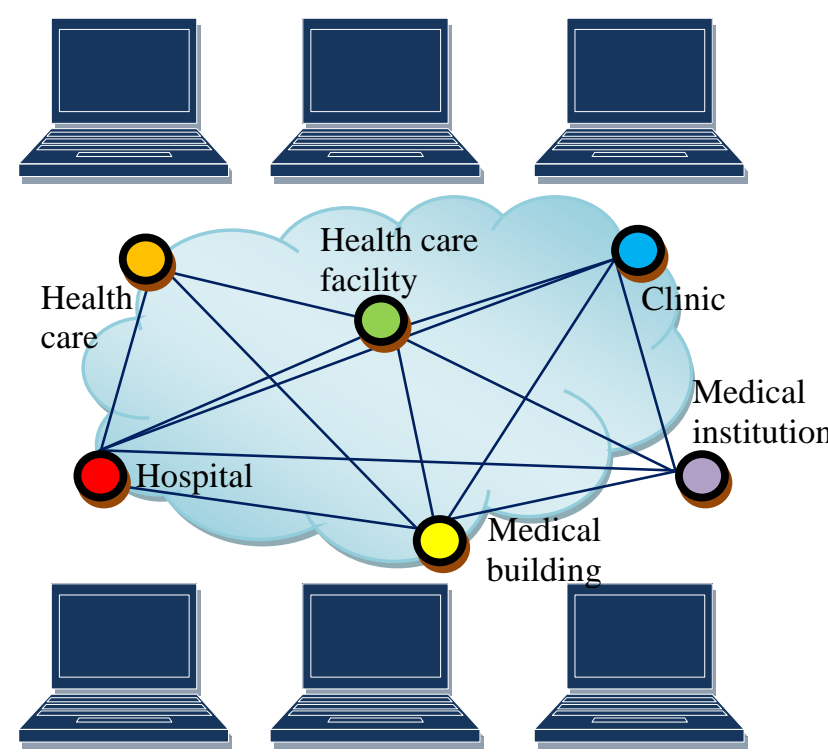

Group of Computer

Fig 4. Mapping in the cloud

Ontology mapping can support systems to share different conceptualizations, different terminologies, and different meanings without any problem in differences. Through this reason, we can say that ontology is a good way to solve the problem of differences, especially in data heterogeneity to simplify the search process. The effectiveness of a search process is give a contibute to energy saving. Next section will describe more deatil about semantic integration process.

\section{IMPLEMENTATION}

In this section we will discuss about how to implement semantic integration trough several ontologies with Protege ${ }^{1}$. There are five (5) ontologies in same domain - "Health", each ontology refer a "building of treatment" with different terms e.g. Ontology1 : Hospital, Ontology2 : Medical Institution, Ontology3 : Infirmary, Ontology4 : Medical_building and Ontology5 : Healthcare_facility. Using ontology mapping we can make correspond between classes (concepts) in two or more ontology. Fig 5 shows correspond between classes in OWL visualization. OWL is a language for create ontologies to the web. OWL was designed for processing information and to provide a common way to process the content of web information. See Fig 5. Hospital is corresponding to Infirmary, Medical building, Health facility, Healthcare facility, clinic, and medical institution; or it can be write that Infirmary is-a hospital, medical building is-a hospital, health facility is-a hospital, healthcare facility is-a hospital, clinic is-a hospital, medical institution is-a hospital, and extended care facility is-a hospital. Relation process should be implementing as a complete graph, if Hospital is corresponding to Infirmary so

\footnotetext{
${ }^{1}$ http://protegewiki.stanford.edu/wiki/Protege4GettingStarted
} 
Infirmary is corresponding to Hospital, and Infirmary also corresponding to Medical building, Health facility, Healthcare facility, clinic, andmedical institution;
SELECT ?Health_Building ?type

WHERE \{ ?Health̄Building :Type ?value.

FILTER (?value= 'hospital'). \}

or with

FILTER (?value= 'Medical building').

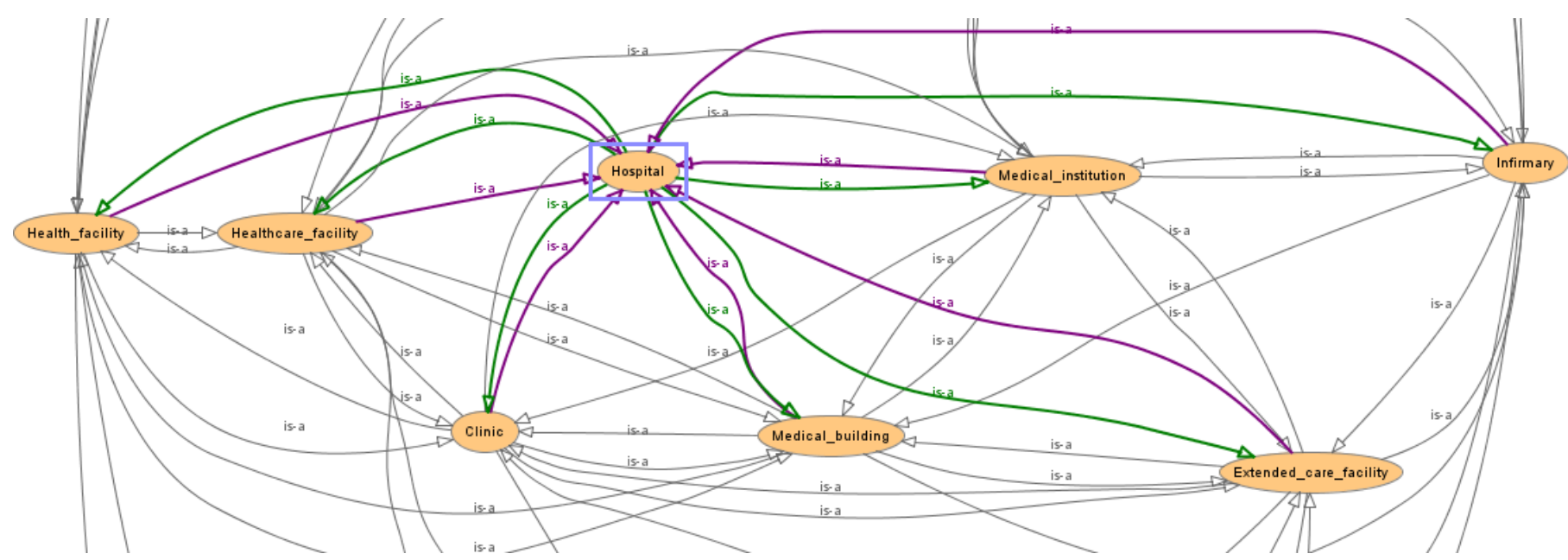

Fig 5. Ontology web language (OWL) visualization in protégé

Individual name of Hospital "Bethesda" shows in RDF syntax below:

<owl: NamedIndividual

rdf: about="\&Ontologies1; Bethesda">

<rdf:type rdf:resource="\&Ontologies1; Hospital"/>

$<$ rdf:type

rdf:resource="Ontologies 2 ; Medical institution"/>

<rdf:type rdf:resource="\&Ontology $\overline{3}$; Infirmary" />

$<$ rdf: type

rdf: resource="\&Ontology4; Medical building"/>

$<$ rdf:type

rdf: resource="\&Ontology5; Health_facility"/>

$<$ rdf:type <rdfs:label

xml: lang="en">Bethesda</rdfs: label>

$<$ Name rdf:datatype="\&xsd; string" $>$ Bethesda $</$ Name $>$ $</$ owl: NamedIndividual>

Ontologies1 refer "Bethesda" as a hospital and Ontologiest5 refer "Bethesda" as a Medical Building. Hospital is corresponding to Medical building and also Medical building is corresponding to Hospital, so user will do searching with both perceptions.

We use a SPARQL ${ }^{2}$ as a graph matching query language to express queries across different data sources.

Prefix:<http://www.semanticweb.org/user/ontologiesl\# $>$

PREFIX rdf: <http://www.w3.org/1999/02/22-rdfsyntax-ns\#>

PREFIX owl: <http://www.w3.org/2002/07/owl\#>

PREFIX xsd: <http://www.w3.org/2001/XMLSchema\#>

PREFIX rdfs: <http://www.w3.org/2000/01/rdf-schema\#>

${ }^{2}$ http://www.w3.org/TR/rdf-sparql-query/
The SPARQL query will refer the same result : Panti rapih, Bethesda and Sarjito as a building : Hospital or as aedical building. (See Fig 6).

\begin{tabular}{ll}
\hline & \multicolumn{1}{c}{ Health_Building } \\
\hline Panti_Rapih & \\
Sarjito & \\
Bethesda &
\end{tabular}

Fig 6 SPARQL result

Semantic engine process with ontology allows users to use their knowledge and perception in their searching activities. User can refer the same object with different term, such as: Bethesda is-a Hospital or Bethesda is-a Medical Building.

\section{DISCUSSIONS}

Green computing inspiring organizations to re-evaluate the use of IT resources. They need to find new ways to increase the energy efficiency. One of the efforts is to improve energy efficiency in the computing process especially search process. One of the major problems in the search process is to make the system can understand the perception that they are referring to. They should be able to make system can think like humans. In order to bring an expected result, they need to be organize data and information in a better way.

Various approaches like Ontology and Semantic Web has been created for better searching on the web. Ontology can be used to process knowledge properly. Semantic engine process with ontology allows users to use their knowledge and 
perception in their searching activities. As discussed in the previous section; user can refer the same object with different term, such as: Bethesda is-a Hospital or Bethesda is-a Medical Building, and machines can understand user perceptions.

Base on this reason in this paper we try to propose an ontology for discovering user needs especially to realize a better search engine. Ontologies can help the machine (search engine) realize the definition of the perception in the metadata. Thing we have done is combine/ integrate several ontologies and achieve general views of the users. Ontologies are used in obtaining results according to the user query and reasoning used to meet the user's needs. Ontology can make a seaching process more efficient and effective.

We can say that ontology can support a green computing main objective especially related to the issue making an intelligent search engine. This project is just the beginning project of big ideas about the role of ontology in the area of green computing. Our future work is about green ontology to support a cost reduction in organizations.

\section{CONCLUSIONS}

In this paper we try to managing knowledge by using an ontology integration as a process to create a new ontology (Global ontology - Common Ontology) to simplify the search process. On the other hand, green computing refers to the systems efficiently and effectively. Hence in this paper we try to collaborate between these two research areas, because if a very large amount of data are managed and distributed properly, user will need a short time to do a search process. The longer the search is done, the more energy is used.

\section{ACKNOWLEDGMENT}

We would like to acknowledge the support of the Erasmus Mundus EuroAsia program for the research foundation of this research, and also to acknowledge Universidade do Minho and Universitas Gadjah Mada for the collaboration

\section{REFERENCES}

[1] S. Murugesan, "Harnessing green IT: Principles and practices," It Prof., vol. 10, no. 1, pp. 24-33, 2008.

[2] A. S. Tanenbaum and M. Van Steen, Distributed systems, vol. 2. Prentice Hall, 2002.

[3] D. Theodoratos, "Semantic integration and querying of heterogeneous data sources using a hypergraph data model," Adv. Databases, pp. 798-805, 2002.

[4] Y. Xue, H. Ghenniwa, and W. Shen, "Ontological viewdriven semantic integration in collaborative networks," Leveraging Knowl. Innov. Collab. Networks, pp. 311318, 2009.

[5] A. P. Sheth, "0 Changing Focus on Interoperability in Information Systems: From System, Syntax, Structure to Semantics," Interoperating Geogr. Inf. Syst., pp. 529, 1998
[6] N. Guarino, Formal ontology in information systems. Citeseer, 1998.

[7] J. F. Sowa, Knowledge representation: logical, philosophical, and computational foundations, vol. 594. MIT Press, 2000.

[8] C. Diamantini, D. Potena, and W. W. Smari, "Collaborative knowledge discovery in databases: A knowledge exchange perspective," in Fall Symposium on Semantic Web for Collaborative Knowledge Acquisition. AAAI, Arlington, VA: IEEE Computer Society, 2006, pp. 24-31.

[9] N. Choucri, Mapping sustainability. Springer, 2007.

[10] K. Knight and S. K. Luk, "Building a large-scale knowledge base for machine translation," in Proceedings of the National Conference on Artificial Intelligence, 1994, pp. 773-773.

[11] T. Kumazawa, O. Saito, K. Kozaki, T. Matsui, and R. Mizoguchi, "Toward knowledge structuring of sustainability science based on ontology engineering," Sustain. Sci., vol. 4, no. 1, pp. 99-116, 2009.

[12] E. Jiménez-Ruiz, B. C. Grau, I. Horrocks, and R. Berlanga, "Ontology integration using mappings: Towards getting the right logical consequences," in The Semantic Web: Research and Applications, Springer, 2009, pp. 173-187.

[13] B. C. Grau, B. Parsia, and E. Sirin, "Ontology integration using $\varepsilon$-connections," in Modular Ontologies, Springer, 2009, pp. 293-320.

[14] H. Wache, T. Voegele, U. Visser, H. Stuckenschmidt, G. Schuster, H. Neumann, and S. Hlübner, "Ontologybased integration of information-a survey of existing approaches," in IJCAI-01 workshop: ontologies and information sharing, 2001, vol. 2001, pp. 108-117.

[15] N. F. Noy and M. A. Musen, "Algorithm and tool for automated ontology merging and alignment," in Proceedings of the 17th National Conference on Artificial Intelligence (AAAI-00). Available as SMI technical report SMI-2000-0831, 2000.

[16] N. F. Noy and M. A. Musen, "The PROMPT suite: interactive tools for ontology merging and mapping," Int. J. Hum.-Comput. Stud., vol. 59, no. 6, pp. 9831024, 2003.

[17] W. Binder and N. Suri, "Green computing: Energy consumption optimized service hosting," in SOFSEM 2009: Theory and Practice of Computer Science, Springer, 2009, pp. 117-128.

[18] "Sustainable Computing: It's Not Easy Being Green," Computerworld, 20-Mar-2006. [Online]. Available: http://www.computerworld.com/s/article/109629/Sustai nable_Computing_It_s_Not_Easy_Being_Green.

[Accessed: 16-Jun-2013]. 